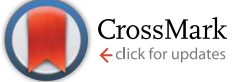

Cite this: RSC Adv., 2016, 6, 44212

Received 9th March 2016 Accepted 27th April 2016

DOI: $10.1039 / c 6 r a 06281 d$

www.rsc.org/advances

\section{Dynamically controlled construction of microstructures based on photo-induced phase transition of a spirobenzopyran-modified polymer solution $\uparrow$}

\author{
Kousuke Moriyama, Kimio Sumaru, ${ }^{*}$ Toshiyuki Takagi, Taku Satoh \\ and Toshiyuki Kanamori
}

Dynamically controlled construction of microstructures was demonstrated using a spirobenzopyran-containing poly $(\mathrm{N}$-isopropylacrylamide). Under patterned light irradiation, the polymer accumulated at the irradiated area from a uniformly dissolved state and formed micro-patterned structures based on phase transition. Furthermore the microstructures were collapsed due to redissolution of the precipitated polymer when light irradiation was stopped.

Including development of biomimetic materials, methodology learned from biological systems has been actively examined in the field of engineering, and has already achieved many successful outcomes. ${ }^{1-5}$ In addition to the accomplished functions, also the process in the constitution of the biological systems themselves is worth examining for its important features, i.e. dynamics. In the process of biological development, for example, a destructive process (i.e. apoptosis) plays an important role as well as a constructive process through the cellular division, to form intricate structures of tissues and organs in a rational manner without wasting the resources. ${ }^{6,7}$ Although self organization under non-equilibrium state has been utilized to form highly ordered structures, ${ }^{\mathbf{8} 9}$ artificial implementation of dynamic processes including destructive mechanism has hardly been reported until now.

Inspired by such a dynamic process of constructing biological systems, here we propose a new type of molecular manipulation using a photo-responsive polymer of which aggregation is controlled by light irradiation. Previously we synthesized poly( $N$-isopropyl acrylamide) (pNIPAAm) modified with spirobenzopyran (SPBs) moieties (p(SPB-NIPAAm)). ${ }^{\mathbf{1 0 - 1 2}}$ SPB,

Biotechnology Research Institute for Drug Discovery, National Institute of Advanced Industrial Science and Technology (AIST), Tsukuba Center 5th, 1-1-1 Higashi, Tsukuba, Ibaraki 305-8565, Japan. E-mail: k.sumaru@aist.go.jp; Fax: +81-29-8616278; Tel: +81-29-861-6373

$\dagger$ Electronic supplementary information (ESI) available: Experimental procedure, irradiated patterns, additional data and supplemental movie file. See DOI: 10.1039/c6ra06281d which is one of the most known photochromic molecules, induces changes in the molecular configuration by visible-light irradiation. ${ }^{13}$ Especially in acidic $(\mathrm{pH} \sim 3)$ aqueous system, all the SPBs are in protonated ring-opening form ( $\mathrm{McH}$ form), which is cationic and hydrophilic. On the other hand, most of them isomerize into hydrophobic ring-closed form ( $\mathrm{Sp}$ form) upon blue light irradiation. ${ }^{11}$ After stopping the irradiation, all the Sps recover to former $\mathrm{McH}$ gradually. Due to the properties of SPB and pNIPAAm, the phase transition of p(SPB-NIPAAm) under aqueous conditions is controlled by the irradiation of visible-light as well as by changing the temperature; the phase transition temperature decreased drastically by visible-light irradiation.

Here, we demonstrated the dynamic control of dissolution and reconstruction of the microstructures based on phase transition of photo-responsive polymer. About the structure of the polymer, we previously reported that substitution of methoxy group at 8 -position of SPB ( $o$-MeO-SPB) accelerates the spontaneous recovery from $\mathrm{Sp}$ to $\mathrm{McH}$ form after stopping irradiation compared with an unsubstituted SPB. ${ }^{14}$ Furthermore, we found that photo-responsive hydrogel composed of pNIPAAm modified with $o$-MeO-SPB moieties ( $\mathrm{p}(o-\mathrm{MeO}-\mathrm{SPB}-\mathrm{NIPAAm}))$ exhibits rapid photo-induced shrinking and fast reswelling in the dark. ${ }^{15}$ Based on these results, we used $\mathrm{p}(o$-MeO-SPB-NIPAAm $)$ as a photo-controllable building block. Under light irradiation, the dehydration of $\mathrm{p}(o$-MeO-SPB-NIPAAm $)$ is induced and the precipitation is obtained at light irradiated area from uniformly dissolved state. On the other hand, the precipitated polymer starts to redissolve into solution when the light irradiation is stopped (Fig. 1). $\mathrm{p}(o$-MeO-SPB-NIPAAm $)$ was prepared by radical copolymerization. The content of $o$-MeO-SPB units in the polymer was determined to be $c a .2 \mathrm{~mol} \%$ by comparing the integrals of signals in ${ }^{1} \mathrm{H}-\mathrm{NMR}$ spectra at $\delta 5.73$ and $\delta 6.63$ for $o$-MeO-SPB, and $\delta 3.97$ for pNIPAAm (Fig. S1†).

First, we confirmed the isomerization of SPB moieties of the polymer. Absorption spectra of $0.05 \mathrm{wt} \% \mathrm{p}(o-\mathrm{MeO}-\mathrm{SPB}-\mathrm{NIPAAm})$ in $1 \mathrm{mM} \mathrm{HCl}$ aqueous solution was measured using UV-vis spectrometer at $0.5{ }^{\circ} \mathrm{C}$ to prevent phase transition of polymer. Before 
(A)

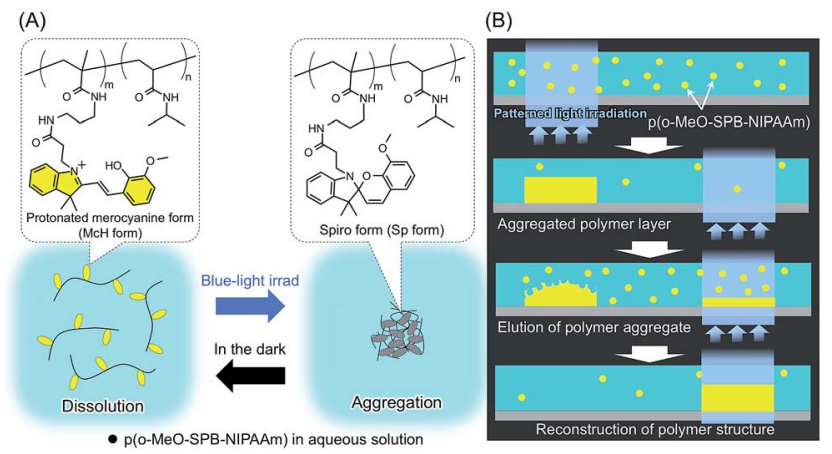

Fig. 1 (A) Isomerization of SPBs and associated behaviour of $\mathrm{p}(\mathrm{O}$ MeO-SPB-NIPAAm) in aqueous solution and (B) proposed system for polymer manipulation by light irradiation.

light irradiation, polymer solution exhibited intense absorbance at around $407 \mathrm{~nm}$ derived from $\mathrm{McH}$ form of SPB moieties, whereas the absorbance decreased drastically after $3 \mathrm{~min}$ of blue-light (wavelength: $436 \mathrm{~nm}$ ) irradiation (Fig. S2(A)†). This result suggested that isomerization from $\mathrm{McH}$ to $\mathrm{Sp}$ form proceeded rapidly in acidic condition by the light irradiation. On the other hand, the absorbance started to increase when light irradiation was stopped and recovered to the former state in ca. $20 \mathrm{~min}$ (Fig. S2(B) $\dagger$ ), indicating the gradual back isomerization from $\mathrm{Sp}$ to $\mathrm{McH}$ form as observed in our previous report. ${ }^{14} \mathrm{Next}$, photoinduced phase transition of $\mathrm{p}(o-\mathrm{MeO}-\mathrm{SPB}-\mathrm{NIPAAm})$ in aqueous $\mathrm{HCl}$ solution was investigated by measuring the turbidity of polymer solution. The temperature-dependent increases of the turbidity at $700 \mathrm{~nm}$ without and with light irradiation were observed above $34{ }^{\circ} \mathrm{C}$ and $26^{\circ} \mathrm{C}$, respectively (Fig. S3 $\dagger$ ), indicating that the phase transition temperature was decreased significantly by blue light. Based on this result, the following experiments were carried out at $30{ }^{\circ} \mathrm{C}$ to induce phase transition by light irradiation. Time dependence measurement was also evaluated. After 3 min of blue-light irradiation, the polymer solution (concentrations of $\mathrm{p}(o$-MeO-SPB-NIPAAm) and $\mathrm{HCl}$ were $0.2 \mathrm{wt} \%$ and $4 \mathrm{mM}$, respectively) turned to be very opaque, indicating drastic dehydration of $\mathrm{p}(o-\mathrm{MeO}-\mathrm{SPB}-\mathrm{NIPAAm})$ (Fig. 2(A)). Furthermore, when
(A)

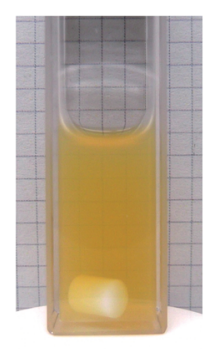

(B)

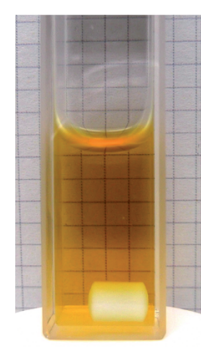

(C)

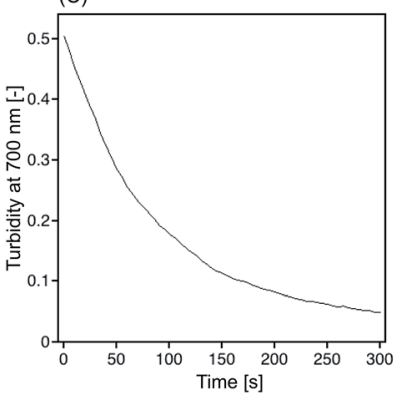

Fig. 2 Phase transition of $\mathrm{p}(\mathrm{O}-\mathrm{MeO}-\mathrm{SPB}-\mathrm{NIPAAm})$ by blue light irradiation. Photographs of $\mathrm{p}(\mathrm{o}-\mathrm{MeO}-\mathrm{SPB}-\mathrm{NIPAAm})$ solution (A) just after 3 min of light irradiation and (B) 5 min after the photo irradiation was stopped. (C) Temporally change in the turbidity at $700 \mathrm{~nm}$ of $\mathrm{p}$ (oMeO-SPB-NIPAAm) solution after 3 min irradiation was stopped. light irradiation was stopped, the turbidity at $700 \mathrm{~nm}$ decreased dramatically in 5 min (Fig. 2(C)) and the solution became clear (Fig. 2(B)).

Photo-induced phase transition was thus observed in the aqueous acidic condition, photo-control of construction and destruction of micro-patterned structures composed of aggregated polymer was investigated using computer-controlled photo-irradiation system in the following procedure. A $\mathrm{p}(o$-MeO-SPB-NIPAAm) solution (concentrations of $\mathrm{p}(o-\mathrm{MeO}-$ SPB-NIPAAm) and $\mathrm{HCl}$ were $0.2 \mathrm{wt} \%$ and $4 \mathrm{mM}$, respectively) was enclosed in a micro chamber composed of glass plates and poly(dimethylsiloxane) sheet with a small hole (diameter and height were $1 \mathrm{~mm}$ and $0.5 \mathrm{~mm}$, respectively). The micro chamber was placed on the microscope stage of photo irradiation system with a $10 \times$ lens with numerical aperture of 0.3 (UPlanFLN $10 \times$, Olympus). We first evaluated the influence of the focal position on the accumulation of polymer at the irradiation area. A stripe-patterned light of $10 \mu \mathrm{m}$ width at $20 \mu \mathrm{m}$ intervals (Fig. S4(B)†) was irradiated at several focal positions between $50 \mu \mathrm{m}$ above and below the chamber bottom. With a light irradiation focused at the chamber bottom, change in the brightness was observed at the irradiated area in phase contrast images (Fig. S5(C) $\dagger$ ), indicating the $\mathrm{p}(o-\mathrm{MeO}-\mathrm{SPB}-\mathrm{NIPAAm})$ was accumulated by dehydration with blue light. The width of striped aggregates of $\mathrm{p}(o$-MeO-SPB-NIPAAm) were $c a .10 \mu \mathrm{m}$, suggesting that the accumulation of polymer proceeded accurately reflecting the irradiated pattern. On the other hand, the accumulation of polymer was rarely observed under the conditions with the focal position below the chamber bottom (Fig. S5(A and B) $\dagger$ ). Since the light intensity decreased with distance from the focal position in the irradiation condition, the result suggested that the isomerization of SPB from $\mathrm{McH}$ to Sp form proceeded insufficiently to induce the polymer accumulation on the chamber bottom. With the focal position above the chamber bottom, blurred patterns were observed (Fig. S5(D and F) $\dagger$ ). These results indicated that the accumulation of polymer can be fully controlled by the micro-patterned irradiation when the focal position is set at the chamber bottom precisely.

Subsequently, we investigated the time dependence of light irradiation on the polymer accumulation in the condition that the chamber bottom was set in focus. Another striped pattern without a line in the center (Fig. S4(A) †) was used. Fig. 3 shows the phase contrast microscopic images of the accumulated polymer. Within 2 min of irradiation, $\mathrm{p}(o-\mathrm{MeO}-\mathrm{SPB}-\mathrm{NIPAAm})$ started to accumulate at irradiated area (Fig. 3(A and B)). With subsequent irradiation, amount of aggregated polymer increased gradually in 220 min (Fig. 3(C-E)). Since phase transition of polymer occurs within several minutes (Fig. 2(A) and $\mathrm{S} 2(\mathrm{~A}) \dagger)$, this result suggested that the diffusion of polymer in the solution is rate-limiting step for the accumulation under the micro-patterned photo-irradiation. After $220 \mathrm{~min}$ of irradiation of the defective stripe-patterned light, the complete stripe-patterned light (Fig. S4(B) $\dagger$ ) was irradiated for $20 \mathrm{~min}$. The additional accumulation of polymer on the central line was rarely observed (Fig. 3(F)), though the polymer was accumulated clearly after the first photo-irradiation for $20 \mathrm{~min}$ (Fig. 3(C)). This result strongly suggested that the most of $\mathrm{p}(o-\mathrm{MeO}-\mathrm{SPB}-$ 

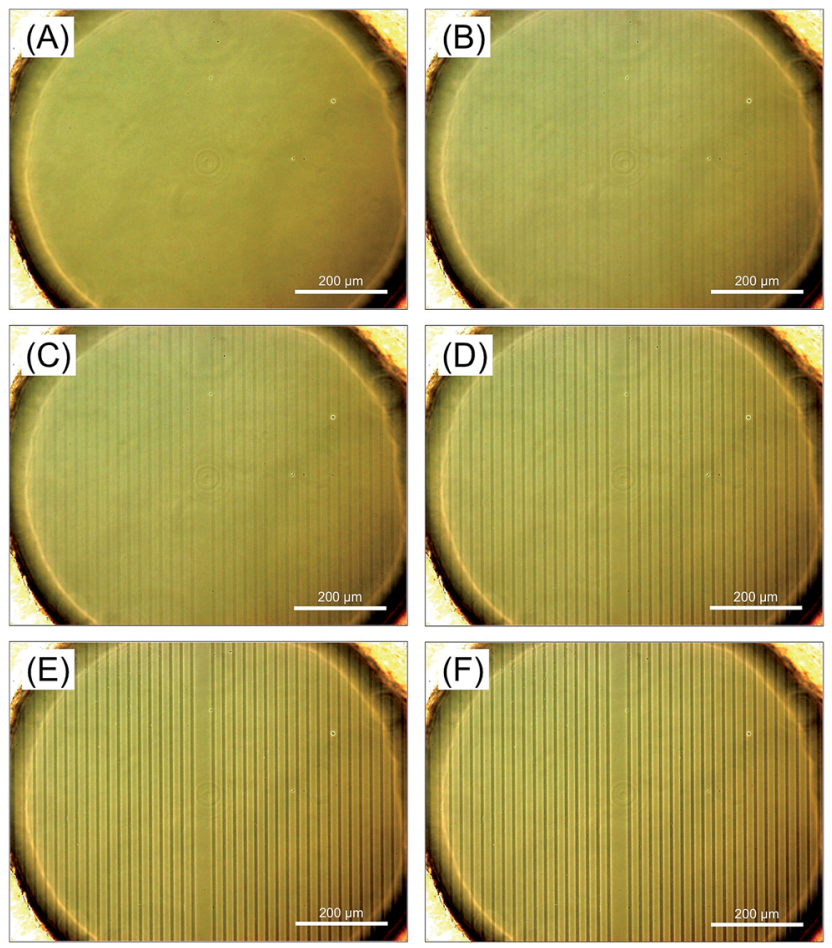

Fig. 3 Phase contrast images of the micro chamber containing $\mathrm{p}(\mathrm{o}$ MeO-SPB-NIPAAm) solution (A) before and (B-F) after irradiation of stripe-patterned light. Defective stripe-patterned light (Fig. S4(A) †) was irradiated for (B) $2 \mathrm{~min},(C) 20 \mathrm{~min},(D) 120 \mathrm{~min}$, and (E) $220 \mathrm{~min}$ and complete stripe-patterned light (Fig. S4(B) †) was continuously irradiated for (F) $20 \mathrm{~min}$.

NIPAAm) in the system was accumulated to form the microstructures on the chamber bottom by the first photo-irradiation for $220 \mathrm{~min}$, and the dissolved polymer was depleted in the solution.

According to the optical theory, change in the local brightness in the phase contrast microscopic image is proportional to the relative contrast of the light path length. ${ }^{16}$ This indicates that change in the brightness $(\Delta b)$ between light irradiated and un-irradiated area is proportional to the areal density of accumulated polymer under the conditions that the geometry of light irradiation region is the same. In order to further discuss the polymer accumulation, we estimated the relationship between the areal density of accumulated polymer and $\Delta b$. The blue light with the defective striped patterns with the same line width $(10 \mu \mathrm{m})$ but with different intervals $(20-50 \mu \mathrm{m})$, i.e. geometry of irradiated region was the same and total irradiated area was varied, (Fig. S4(A, C, E and G) $\dagger$ ) were irradiated to $0.2 \mathrm{wt} \% \mathrm{p}(o-\mathrm{MeO}-\mathrm{SPB}-\mathrm{NIPAAm})$ solution for $220 \mathrm{~min}$ to deplete the dissolved polymer as described above (Fig. S6 $\dagger$ ). In the obtained phase contrast images, $\Delta b$ was calculated and proportional relationship between the inverse of the irradiated area and $\Delta b$ was observed (Fig. S7 $\dagger$ ). This result also supports that most of the polymer was accumulated at irradiated area by prolonged photo-irradiation as described above (Fig. 3(E and F)). Based on these results, areal density of accumulated polymer can be estimated from the phase contrast images under certain experimental conditions (Fig. S7 $\dagger$ ).
Finally, we demonstrated the dynamic control of accumulation of $\mathrm{p}(o$-MeO-SPB-NIPAAm $)$ by continuous photo-irradiation using various types of patterns (Fig. S8, $\uparrow$ and 4 , and supplemental movie). After the first photo-irradiation, accumulation of polymers in a chinese character '光' (light) was observed at left and right sides according to the irradiated pattern (Fig. S8(A) $\dagger$ and 4(A and B)). With the second patterned light irradiation non-containing the first irradiated area (Fig. S8(B)†), the micro-patterned polymer aggregates were formed in the newly irradiated areas. On the other hand, the aggregates formed in the first irradiation disappeared (Fig. 4(C)). This result indicated the formation and the maintenance of micropatterned structures was temporary only under the photoirradiation. Furthermore, the destruction of the arbitrary microstructures was achieved at desired time. After second irradiation, we appended the irradiation area in the center of the chamber (Fig. S8(C) †). However, further accumulation of polymer was not observed (Fig. 4(D)), indicating most of the polymer in the system was used for the formation of microstructures without wasting as described above. After stopping the irradiation excepted the chinese character, polymer accumulation was observed there (Fig. S8(D) $\dagger$ and 4(E)). This result also showed an important feature of proposed system; the polymers as building blocks of micro-patterned structures were transported from the previously irradiated areas to the other distant area via dissolved state. Before reaching saturation, we irradiated other different pattern including the previously
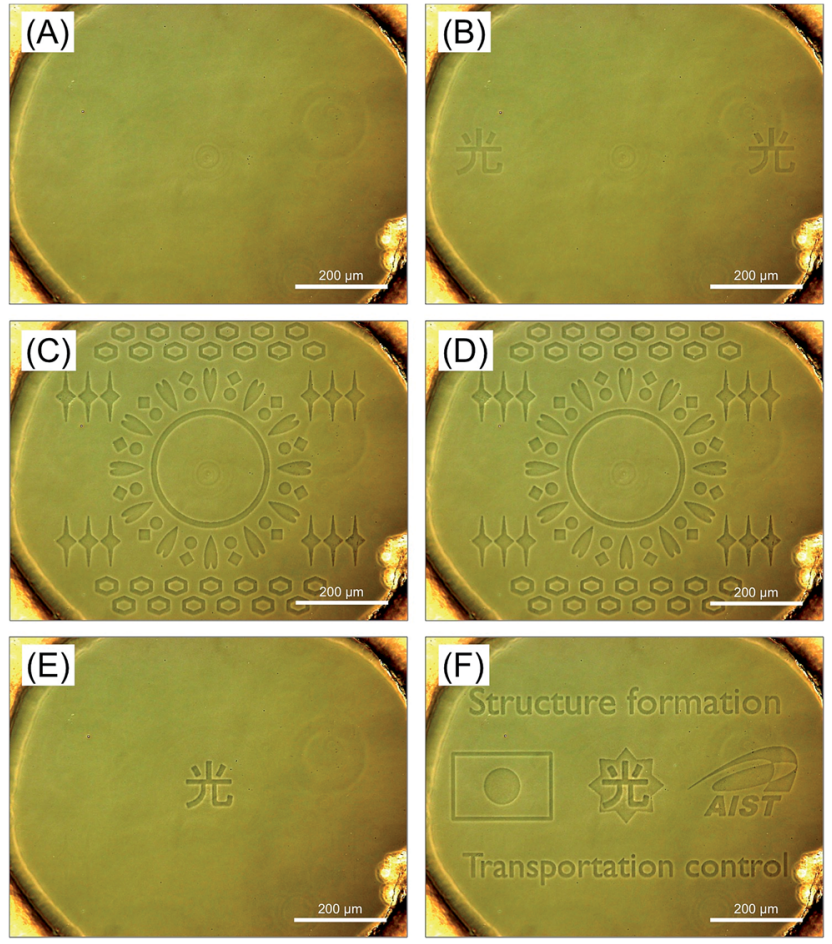

Fig. 4 Phase contrast images of the micro chamber containing ploMeO-SPB-NIPAAm) solution (A) before and (B-F) after irradiation of various types of patterns. Continuous irradiation was performed for (B) $10 \mathrm{~min}$ (Fig. S8(A)†), (C) $60 \mathrm{~min}$ (Fig. S $8(B) \dagger$ ), (D) $10 \mathrm{~min}$ (Fig. S $(C) \dagger$ ), (E) 30 min (Fig. S8(D)†) and (F) 40 min (Fig. S $8(E) \dagger)$. 
irradiated area in the center (Fig. S8(E) $\dagger$ and 4(F)). As suggested from the results shown above, polymer accumulation was observed in the newly irradiated area. In the area including the previously irradiated pattern, micro-patterned structure with a step was observed (a chinese character and a star-shaped pattern show upper and lower steps, respectively) (center in Fig. 4(F)). This result suggested that three-dimensional polymer-based microstructures can be formed by step-wise photo-irradiation successively.

\section{Conclusions}

We synthesized a photo-responsive polymer with pNIPAAm main chain and $o$-MeO-SPB moieties at side chains, which precipitated only under blue light irradiation in acidic aqueous system. Using the polymer and micro-patterned light irradiation, we demonstrated dynamic control of polymer accumulation in arbitrary areas to build and scrap micro-patterned structures. In a closed chamber with the size of $1 \mathrm{~mm}$, the building blocks were provided from uniformly dissolved state and integrated into the structures without being wasted. The structure was destructed by stopping the irradiation and the building blocks became dissolved for subsequent formation of the microstructures at desired area. The polymers were transported in the solution through convection and diffusion to newly irradiated areas. Although the microstructures implemented here were basically two-dimensional, we believe that the proposed scheme of spatio-temporal control provides any clue to meet requirements for realizing highly functional microsystems like biosystems.

\section{Acknowledgements}

This research was supported by the KAKENHI Grant-in-Aid for Scientific Research B $(20350110,25282148)$ from Japan Society of Promotion of Science (JSPS).

\section{Notes and references}

1 N. Zhao, Z. Wang, C. Cai, H. Shen, F. Y. Liang, D. Wang, C. Y. Wang, T. Zhu, J. Guo, Y. X. Wang, X. F. Liu, C. T. Duan, H. Wang, Y. Z. Mao, X. Jia, H. X. Dong, X. L. Zhang and J. Xu, Adv. Mater., 2014, 26, 6994-7017.

2 K. S. Liu and L. Jiang, Nano Today, 2011, 6, 155-175.

3 F. Xia and L. Jiang, Adv. Mater., 2008, 20, 2842-2858.

4 A. R. Studart, Angew. Chem., Int. Ed., 2015, 54, 3400-3416.

5 Q. Zhao, D. W. Lee, B. K. Ahn, S. Seo, Y. Kaufman, J. N. Israelachvili and J. H. Waite, Nat. Mater., 2016, 15, 407-413.

6 A. G. Renehan, S. P. Bach and C. S. Potten, Scand. J. Gastroenterol., 2001, 15, 166-176.

7 P. E. Bourgine, B. E. Pippenger, A. Todorov, L. Tchang and I. Martin, Biomaterials, 2013, 34, 6099-6108.

8 N. Maruyama, T. Koito, J. Nishida, T. Sawadaishi, X. Cieren, K. Ijiro, O. Karthaus and M. Shimomura, Thin Solid Films, 1998, 327, 854-856.

9 M. Shimomura and T. Sawadaishi, Curr. Opin. Colloid Interface Sci., 2001, 6, 11-16.

10 K. Sumaru, M. Kameda, T. Kanamori and T. Shinbo, Macromolecules, 2004, 37, 4949-4955.

11 K. Sumaru, M. Kameda, T. Kanamori and T. Shinbo, Macromolecules, 2004, 37, 7854-7856.

12 K. Sumaru, T. Takagi, T. Satoh and T. Kanamori, J. Photochem. Photobiol., A, 2013, 261, 46-52.

13 R. Klajn, Chem. Soc. Rev., 2014, 43, 148-184.

14 T. Satoh, K. Sumaru, T. Takagi, K. Takai and T. Kanamori, Phys. Chem. Chem. Phys., 2011, 13, 7322-7329.

15 T. Satoh, K. Sumaru, T. Takagi and T. Kanamori, Soft Matter, 2011, 7, 8030-8034.

16 C. A. Mack, Proc. Soc. Photo-Opt. Instrum. Eng., 1993, 1927, 512-520. 